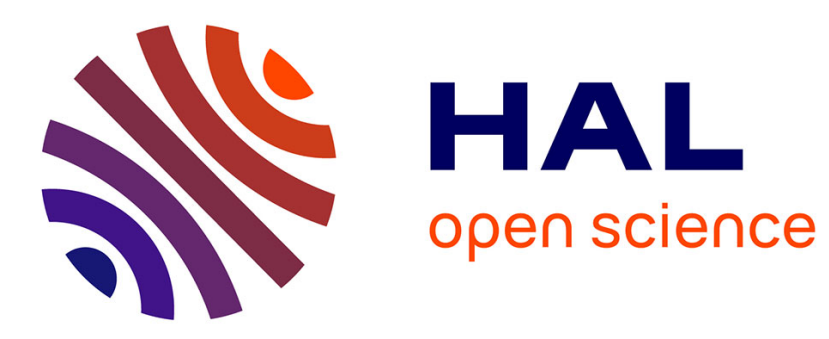

\title{
Société politique et société religieuse
}

Michel Bourdeau

\section{To cite this version:}

Michel Bourdeau. Société politique et société religieuse: essai de politique positive. Commentaire, 2020, 169. halshs-00941540

\section{HAL Id: halshs-00941540 \\ https://shs.hal.science/halshs-00941540}

Submitted on 6 Feb 2014

HAL is a multi-disciplinary open access archive for the deposit and dissemination of scientific research documents, whether they are published or not. The documents may come from teaching and research institutions in France or abroad, or from public or private research centers.
L'archive ouverte pluridisciplinaire HAL, est destinée au dépôt et à la diffusion de documents scientifiques de niveau recherche, publiés ou non, émanant des établissements d'enseignement et de recherche français ou étrangers, des laboratoires publics ou privés. 


\title{
Société politique et société religieuse \\ Essai de politique positive.
}

\author{
Michel Bourdeau, \\ IHPST (CNRS-Paris1-ENS)
}

\begin{abstract}
Une société de sociétés politiques ne saurait être elle-même une société politique; ce ne peut être qu'une société religieuse. [...] Être une « sociolâtrie », c'est-à-dire se faire accepter comme le seul lien social possible au-delà de la cité, sera désormais l'essence de la religion . E. Gilson
\end{abstract}

Un oubli plus total que celui dans lequel est tombé la politique positive est difficile à imaginer et l'on en chercherait en vain le moindre écho dans les débats contemporains. Il n'en a pas toujours été ainsi et, comme le rappelait Aron dans cette même revue ${ }^{1}$, entre les deux guerres, les deux maîtres à penser de la jeunesse, Alain et Maurras, se réclamaient l'un et l'autre de Comte. Tout ceci s'est effondré avec la Troisième République sous l'assaut des chars allemands et depuis lors, s'il arrivait encore d'entendre parler de politique positive, c'était presque toujours sous une forme caricaturale. Comte devenait une sorte de théoricien du totalitarisme et l'on ne retenait de lui que ses critiques des droits de l'homme et du régime parlementaire, ou ses éloges de la dictature.

Aujourd'hui, divers indices donnent à penser que cette situation pourrait être en train de changer. Les pages qui suivent se proposent de le montrer sur l'exemple de la religion. L'Europe, avec le traité de Westphalie, la France, avec les lois de 1905, croyaient avoir réglé la question religieuse mais l'actualité est là pour nous rappeler qu'il n'en est rien : les conflits qui déchirent un peu partout la planète ont bien souvent une dimension religieuse et la mondialisation fait qu'ils nous affectent parfois de façon directe. Or la religion fournit un bon exemple de la situation faite à la pensée politique de Comte. La religion de l'Humanité, qu'il avait fondée, n'a jamais compté plus qu'une poignée de fidèles et, pour le discréditer, il suffit de rappeler que l'autoproclamé grand-prêtre s'était fait confectionner un sceau pontifical et était persuadé qu'il finirait par prêcher à Notre-Dame. S'en tenir là, ou même ne considérer, comme on le fait le plus souvent, que la forme qu'il a voulu donner à la religion de 1'Humanité, serait toutefois passer à côté du plus intéressant et le tome deux du Système de politique positive (désormais Système) contient, sur les rapports de l'Église et de l'État, encore appelés société religieuse et société politique, des réflexions d'une grande originalité2. Des deux termes qui composent l'expression société religieuse, le premier importe autant et même plus que le second. Ce à quoi nous sommes invités, c'est à penser la religion d'un point de vue assez systématiquement ignoré, à savoir le point de vue sociologique. La religion sera donc ici caractérisée avant tout comme un type de société, qu'il s'agira de comparer avec d'autres. C'est dire qu'il ne sera à peu près pas question de la religion de l'humanité dans ce qu'elle a de propre, avec son culte, ses dogmes et ses sacrements.

\footnotetext{
${ }^{1}$ R. Aron: “Alain et Maurras”. Commentaire, n 64, 1993-1994, p. 693-703.

2 Il y a plus de cinquante ans, dans Les métamorphoses de la Cité de Dieu (Vrin 1952, réimpression 2005), — dont le dernier chapitre décrit la cité des savants rêvée par Comte E. Gilson a consacré à cette distinction des pages qui ont servi de point de départ à cet essai et qui gagnent encore à être lues
} 
L'exposé procédera en deux temps. Tout d'abord, il conviendra de présenter dans ses grands traits la distinction établie par Comte entre trois types de société : domestique, puis politique et enfin religieuse. Le niveau supérieur s'éclairant par comparaison avec celui qui le précède, il faudra commencer, pour dégager la spécificité de la cité, par dire quelques mots de la famille. Cela permettra de reconnaître au passage ce qu'il y a de bien fondé dans les théories du contrat social : la principale objection adressée au contractualisme, à savoir qu'il ignore le caractère naturel de la vie sociale, tombe en effet dès lors qu'on prend soin de distinguer entre société domestique et société politique. Ces préliminaires une fois posés, nous pourrons examiner les rapports de l'Église et de l'État. Le gouvernement politique issu de la coopération ne tarde pas en effet à montrer ses limites. Une société de sociétés politiques ne saurait être une société politique. D'où la nécessité d'un troisième type de société qui vienne compléter la cité. S'il importe de distinguer soigneusement l'Église et l'État, il importe tout autant de reconnaître la prépondérance de ce dernier. La société politique est la seule à être examinée deux fois, dans son rapport et à la famille et à l'Église.

$\mathrm{Vu}$ les fortes résistances rencontrées par la théorie positive de la religion, il faudra, dans un second temps, examiner plus en détail certains de ses aspects. Si en effet l'adoption du point de vue sociologique entraîne une approche purement terrestre de la religion, il reste encore à mieux déterminer la nature de celle-ci. Pour ce faire, il suffira de s'arrêter sur ses deux principaux caractères : elle est à la fois la plus étendue et la moins complète. Le premier d'entre eux souligne l'importance cruciale des questions de taille. Ce sont elles par exemple qui motivent la séparation de l'Église et de l'État. Contrairement au mouvement qui a prévalu dans les temps modernes et qui a conduit à la création des États nations, les positivistes estiment qu'une domination sans tyrannie ne peut s'exercer qu'à l'intérieur d'un territoire de petite dimension (la Corse ou la Belgique). Sauf à devenir oppressive, toute tentative pour dépasser cette taille demande la constitution d'une société religieuse, seule en mesure de s'étendre à l'ensemble de la planète. Mais cette universalité a un prix. A mesure que le lien social s'étend, il s'affaiblit, et la société religieuse est aussi la moins complète, car elle ne repose que sur un accord intellectuel. Ce qui unit les membres de la société religieuse, ce ne sont pas tant des sentiments ou des pratiques que des croyances communes; et s'il est vrai que le positivisme a toujours affirmé que toute société est fondée sur l'opinion - en ce sens que la vie sociale serait impossible sans un consensus minimum sur les principes qui la régissent-, il a aussi toujours souligné la faiblesse native de notre intelligence.

Les trois sociétés.

Le Système nous invite à distinguer trois types de société : la famille, la cité ou État, l'église. La cité se compose de familles comme l'Église se compose de cités. Que la science sociale doive commencer par une théorie de la famille, il n'y a pas d'enseignement plus constant chez Comte, car c'est par là que la sociologie touche à la biologie. En revanche, on chercherait en vain dans le Cours une quelconque mention de la société religieuse. Ce silence renvoie au fait que ces trois strates ne sont pas seulement superposées mais aussi successives et que, à la différence des deux premières, la société religieuse est pour l'essentiel encore à venir. De là deux difficultés. En s'éloignant du domaine factuel pour s'attacher à une réalité encore en gestation, Comte s'exposait au reproche que Durkheim lui a adressé : sa sociologie manquerait de positivité. Par ailleurs, que la critique soit ou non justifiée, on ne peut contester que l'usage ainsi fait de religieux soit une source de malentendus. Dans l'esprit de beaucoup de nos contemporains, la religion appartient beaucoup plus au passé qu'à l'avenir : s'il est vrai que c'est une des formes les plus anciennes de la vie humaine, le déclin des pratiques religieuses dans les sociétés modernes invite à se demander si les beaux jours de la religion ne seraient pas plutôt derrière elle. Les phénomènes que nous avons l'habitude d'appeler religieux sont donc loin de toujours correspondre à ce que Comte entend par là (comment 
comprendre par exemple qu'il puisse affirmer que l'homme devient de plus en plus religieux ?). En particulier, en raison de ce qu'il appelle « l'antique adhérence de l'église avec l'État $»^{3}$, les diverses Églises qui existent ou ont existé ne sont que des anticipations très imparfaites de la société religieuse à venir.

Société domestique et société politique. Quoique la théorie positive de la famille ne nous concerne pas directement, il y a lieu d'en dire quelques mots car elle permet de mettre en relief ce qu'il y a de spécifique dans la société politique. On connaît l'hostilité de Comte envers ceux pour qui la société aurait comme origine un contrat passé entre les individus. Une telle analyse, estime-t-il, est anarchique et dissout l'existence sociale au lieu de l'expliquer. «Une société n'est donc pas plus décomposable en individus qu'une surface géométrique ne l'est en lignes, ou une ligne en points. La moindre société, savoir la famille, quelquefois réduite à son couple fondamental, constitue donc le véritable élément sociologique » (S. 3, 180). Mais pour comprendre ce qui caractérise la vie sociale proprement dite, à savoir l'appartenance à la société politique, il convient de se tourner vers la division du travail. A la fois principe d'un ordre spontané et condition sine qua non du progrès, celle-ci « constitue, en effet, le caractère fondamental des opérations humaines, quand on s'élève du simple point de vue domestique au vrai point de vue social» (C. $\left.50^{\mathrm{e}} 1 ., 191\right)$. On a certainement prêté beaucoup trop peu d'attention à la fin de cette phrase, pourtant parfaitement explicite : la reconnaissance du caractère fondamental de la division du travail est inséparable de la distinction entre vie domestique et vie sociale.

Cette distinction permet de porter, sur les théories du contrat social, un jugement beaucoup plus nuancé que celui qui est attribué d'ordinaire au fondateur du positivisme. Certes, leurs partisans ont «commis une erreur capitale en attribuant à ce principe la création même de l'état social, puisqu'il est, au contraire, évident que la coopération, bien loin d'avoir pu produire la société, en suppose nécessairement le préalable établissement spontané » (C. $50^{\mathrm{e}}$ 1., 193). La comparaison sociologique de l'homme aux autres animaux montre de façon irrécusable « combien sont pleinement naturelles les principales relations sociales » (C. $48^{\mathrm{e}} 1$, 145). Ceci étant, si l'on s'interroge sur les causes d'une telle erreur, on doit l'imputer «à une confusion radicale entre la vie domestique et la vie sociale, trop ordinaire aux spéculations métaphysiques. Car, en séparant convenablement deux modes d'association aussi différents, cette assertion, soigneusement restreinte à la combinaison la plus compliquée, paraîtrait certainement peu choquante » (C. $\left.50^{\mathrm{e}} 1 ., 192\right)$. La question se pose alors de savoir comment justifier cette distinction, ce qui nous renvoie aux rapports de la sociologie et de la biologie. L'erreur des théoriciens du contrat, pour en revenir à eux, est d'avoir négligé « cette partie élémentaire de la sociologie qui se confond presque avec la biologie intellectuelle et morale » (C. $\left.48^{\mathrm{e}} 1 ., 145\right)$; l'auraient-ils fait qu'ils auraient cessé « de regarder comme factices et arbitraires les liens fondamentaux de la famille humaine, en les retrouvant, avec le même caractère essentiel, chez les animaux, et d'une manière d'autant plus prononcée que l'organisme y devient plus élevé, plus rapproché de l'organisme humain » (Ibid.).

Société politique et société religieuse.

La cité. L'apparition de la société politique coïncide donc avec celle de « cette invariable conciliation de la séparation des travaux avec la coopération des efforts » (C. $\left.50^{\mathrm{e}} 1 ., 191\right)$, en quoi consiste la division du travail. Comte en attribue la découverte à Aristote, qui avait déjà noté que « ce n'est pas entre deux médecins que naît une communauté d'intérêt, mais entre un

\footnotetext{
${ }^{3}$ S. 2, 307. Le Système (abrégé en : S., suivi du numéro du volume) est cité dans l'édition de 1929, 4 vols., au siège de la société positiviste. De même, le Cours de philosophie positive (abrégé en C., suivi du numéro de la leçon) est cité dans la nouvelle édition, en cours de publication aux éditions Hermann.
} 
médecin par exemple et un cultivateur, et d'une manière générale entre des contractants différents et inégaux et qu'il faut pourtant égaliser » (Éthique à Nicomaque, V 8). C'est dans ce contexte que Comte a été amené à examiner les contributions de l'économie à la science sociale. Comme dans le cas des théories du contrat social, on ne retient d'ordinaire que les critiques sévères qu'il lui a adressées mais c'est oublier que c'est par l'économie que le jeune polytechnicien a commencé, quand il était secrétaire de Saint-Simon, et que son admiration pour Adam Smith et l'École Écossaise (Hume, Fergusson) ne s'est jamais démentie, jusque dans le Système. C'est ainsi qu'il accorde aux économistes le mérite d'avoir été les premiers à reconnaître l'existence d'un ordre spontané dans les phénomènes sociaux. Il est étonnant que Hayek, qui fait de la reconnaissance d'un ordre spontané le point de départ de la science sociale, n'ait pas vu que l'auteur du Cours ne pensait pas autrement ${ }^{4}$. Le titre de la $50^{\mathrm{e}}$ leçon est pourtant on ne peut plus explicite : Considérations préliminaires sur la statique sociale ou théorie générale de l'ordre spontané des sociétés humaines. Dans un passage qu'Hayek s'étonne de trouver sous la plume de Comte, celui-ci attribue même, sans la nommer, l'existence de cet ordre spontané à la main invisible (C. $\left.50^{\mathrm{e}} 1 ., 261\right)$. Une fois établie l'existence de cet ordre, la question se pose de savoir quelle attitude adopter à son égard, et c'est là que les deux penseurs divergent. Le positivisme croit possible et souhaitable de modifier cet ordre naturel de façon à lui substituer un ordre artificiel plus conforme à nos désirs. Telle est d'ailleurs la fonction du politique, entendu comme art de gouverner : éviter ou plutôt atténuer les crises et faire en sorte que la marche de la civilisation s'effectue sans trop de heurts. Comte ne se fait d'ailleurs pas d'illusion sur la marge de manœuvre dont dispose l'homme politique : nous ne gouvernons pas les phénomènes à notre guise et l'ordre artificiel n'est jamais que le prolongement de l'ordre naturel.

Si le gouvernement était en mesure de remplir de façon satisfaisante sa tâche, la société religieuse serait superflue. Toutefois, même à supposer que les grands États soient démembrés de façon à rendre possible cette domination sans tyrannie que la politique positive veut instaurer, le pouvoir temporel continuerait à reposer en dernière instance sur la force, laquelle « a toujours besoin d'être d'abord doublement complétée, et ensuite convenablement réglée, pour servir de base durable au gouvernement politique » (S. 2, 301). Tout d'abord, si le pouvoir revient aux caractères énergiques, ceux-ci manquent d'ordinaire de lumières. Or le présent sur lequel s'exerce leur action doit être éclairé par la connaissance du passé et la considération du futur. La force a donc besoin d'un guide spirituel qui l'aide à la prise de décision. Elle a également besoin de ce que Comte appelle une "consécration morale », dans la mesure où « l'ascendant politique résulte, directement ou indirectement, d'un concours plus ou moins volontaire, d'ailleurs actif ou passif, constamment susceptible d'être rompu quand l'harmonie affective devient insuffisante » (S. 2, 302). Enfin, tout pouvoir disposant à l'abus, l'instance chargée de réguler les phénomènes sociaux a besoin à son tour d'être réglée.

La société religieuse. Tel est le triple motif qui justifie la création de la société religieuse. Reste maintenant à préciser à quelles conditions elle peut remplir ces fonctions. Dans le cas présent, on peut négliger les deux premières, les qualités intellectuelles et morales requises relevant du domaine individuel. Tout au plus faut-il souligner que le pouvoir spirituel comprend de toute nécessité une dimension morale : il ne suffit pas d'être compétent, encore faut-il être cru, et pour cela digne de confiance. Concernant la troisième fonction, elle implique que l'Église n'a pas sa fin en elle-même : elle n'existe qu'en vue de la cité, lieu par excellence de la vie sociale. Mais l'Église ne doit pas être simplement distincte de l'État. Elle

\footnotetext{
${ }^{4}$ Voir les critiques qu'il lui adresse dans Counter Revolution of Science (1952). La première partie de l'ouvrage a été traduite par R. Barre (Scientisme et science sociale, Plon, 1953); il est regrettable que la partie historique, qui traite essentiellement de Saint-Simon et de Comte, n'ait pas été traduite.
} 
doit rompre «l'antique adhérence » qui la liait à lui et c'est cette exigence qui appelle la création d'un nouveau type de société qui vient se superposer aux précédents.

A tort ou à raison, il est souvent reproché au christianisme de détourner le croyant de l'icibas : au regard de l'éternelle félicité qui l'attend dans la cité céleste, la vie terrestre n'est plus qu'une vallée de larmes. Le positivisme ne met pas seulement en doute l'existence de cette vie future, il tient que l'extension supérieure de l'Église ne lui confère aucune supériorité et proclame que la place prépondérante revient de façon irrévocable à l'État. "C'est donc à la cité, organe essentiel de la coopération active, qu'il faut surtout rapporter l'homme, mais en la concevant sans cesse comme préparée par la famille, et complétée par l'Église » (S. 2, 341).

Cette subordination n'a pas peu contribué à la longue confusion de l'Église et de l'État, alors pourtant que leur séparation est indispensable si l'on veut que la société religieuse remplisse ses fonctions. Jusqu'il y a peu, elle se distinguait mal de la société politique et, si elle possédait bien une existence propre, celle-ci ne correspond guères à la description que nous en donne Comte. Encore que les grands monothéismes s'en soient en partie affranchi, c'était toujours l'antique adhérence de l'Église à l'État qui prévalait et, si les dieux n'étaient plus les dieux de la cité, en pratique, on continuait à appliquer le principe cujus regio, ejus religio. Dans l'exercice de ses deux premières fonctions, l'Église ne s'accommodait pas trop mal de cette situation. Aucune société politique ne pouvant se passer de guide intellectuel et de consécration morale, ces fonctions trouvent toujours à être satisfaites et guider, comme consacrer, suppose une certaine proximité d'avec le pouvoir. Si les positivistes ont œuvré activement en faveur de la séparation de l'Église et de l'État, toute une partie de ce qu'ils entendaient par là a été perdu de vue par le législateur de 1905, puisqu'ils y voyaient le seul moyen de faire advenir une société religieuse. La clé de cette idée à bien des égards déconcertante se trouve dans la dernière fonction de l'Église. On voit mal en effet comment une instance régulatrice, chargée de lutter contre les abus de pouvoir, pourrait remplir correctement sa fonction sans être indépendante de ce qu'elle doit régler, puisque son office est «à la fois répressif et directeur, quoique toujours consultatif » (S. 2, 308). L'issue des conflits susceptibles de surgir serait connue d'avance si l'une des deux se trouvait dès le départ soumise à l'autre. La meilleure garantie de cette indépendance se trouve dans la plus stricte séparation: la société religieuse peut ainsi suivre librement sa tendance vers l'universalité, une extension supérieure lui assurant une sorte d'exterritorialité qui la met à l'abri des atteintes du pouvoir' 5 .

La société la plus étendue et la moins complète.

Une société planétaire. La distinction entre les trois types de société est liée à des considérations de taille. Tout se passe comme s'il existait des seuils que le mode de fonctionnement de la famille ou de la cité leur interdisait de franchir. L'apparition du monothéisme, quant à lui, a rendu manifeste la tendance à l'universalité de la société religieuse. Mais elle n'est pas seule à vouloir s'étendre à toute la planète et une bonne façon de comprendre la spécificité de cette nouvelle forme de vie sociale consiste à la comparer aux autres tentatives entreprises en ce sens, d'autant qu'elles semblent avoir eu plus de succès. On peut tout d'abord l'opposer aux empires. Chercher à différencier l'Église et l'État par leur extension respective reviendrait à oublier qu'il existe une tendance spontanée des Etats à s'étendre indéfiniment. La réponse a déjà été esquissée plus haut : l'existence des empires est

\footnotetext{
5 On s'étonne de voir Comte critiquer Platon et sa doctrine des philosophes rois; mais pouvoir spirituel ne signifie en aucune façon règne de l'esprit. Bien au contraire, forte du principe que l'esprit n'est pas destiné à régner mais à servir, la seconde philosophie de Comte fait de l'esprit le ministre du cœur. La doctrine platonicienne repose sur la confusion des deux pouvoirs.
} 
contraire à la nature du lien politique. Une domination sans tyrannie exige une proximité entre gouvernants et gouvernés et impose donc des contraintes de taille. Si la tendance évoquée existe bien, elle relève de ces abus de pouvoir que la société religieuse a précisément pour but de combattre.

Tout en concédant à Comte la nécessité et de limiter la taille des Etats-nations et de créer des entités supranationales, on peut encore lui reprocher de s'être lourdement trompé sur la direction dans laquelle cette extension devait se réaliser. A l'évidence, il existe des liens sociaux qui s'étendent bien au-delà des liens politiques, mais ils sont à chercher du côté de l'économie et non de la religion. C'est parce qu'il aurait refusé de reconnaître la spécificité et l'autonomie de la sphère économique que Comte n'aurait pas vu que cette dimension supranationale s'est réalisée dans le marché mondial et non dans la société religieuse. L'objection repose sur une double méprise. Tout d'abord, ce n'est pas parce que, dans ce contexte, Comte ne parle pas du marché mondial qu'il en ignore l'existence. En bon disciple de Saint-Simon, il avait si bien perçu la portée du développement industriel qu'il lui avait fait une place dans la loi des trois états. Celle-ci comporte en effet deux versions, spirituelle et temporelle : le premier état est à la fois théologique et militaire, le dernier, positif et industriel. Le contraste entre sociétés militaires et sociétés industrielles, qui parcourt ainsi toute son œuvre, s'applique notamment au cas présent.

Toutes les cités se trouvaient naturellement rivales dans l'âge militaire, soit en tendant à la fois vers une domination unique, soit en résistant séparément à l'incorporation forcée qui pouvait seule les unir. Au contraire, l'état industriel les fait spontanément converger, en assignant à chacune un but qui peut devenir universel, parce qu'il reste toujours extérieur. La commune exploitation du domaine terrestre comporte, entre les diverses républiques, une répartition d'offices équivalent à celle qui coordonne les différentes classes dont se compose chaque peuple. On reconnaît ainsi que l'activité pacifique conduit spontanément à l'association universelle, seule source de sa systématisation, tandis que l'essor guerrier ne comportait que la société civique ${ }^{6}$.

Il existe donc un lien entre l'avènement de la société religieuse et celui de la société industrielle ; mais, et c'est le second point, objecter à Comte le pouvoir de l'économie à unifier la planète revient à ignorer ce vers quoi il veut attirer notre attention. Ce n'est tout simplement pas dans cette direction qu'il faut chercher la réponse aux problèmes qui appellent la création d'une société religieuse. Bien au contraire, pour renverser une formule célèbre, le marché, ce n'est pas la solution, c'est le problème. On se souviendra en effet que le phénomène constitutif de la société politique n'est autre que la division du travail et que le besoin de régulation sociale s'applique donc à la vie économique. Dès 1822, Comte assignait comme objectif au régulateur d' " adoucir et surtout d'abréger les crises ». On se souviendra encore que l'extension supérieure de la société religieuse ne remet pas en cause la primauté de la cité, et avec elle de la vie économique. Le rapport existant entre marché mondial et société religieuse découle de "la théorie fondamentale de la nature humaine, qui, subordonnant l'existence cérébrale à l'existence corporelle, fait toujours prévaloir l'activité sur l'intelligence et même sur le sentiment» (S. 4, 341). Tout au plus pourra-t-on reprocher à Comte d'avoir laissé en suspens la question de savoir comment on peut à la fois vouloir restreindre la taille des Etats et reconnaître que la vie économique qui en est le substrat tend, elle, à ignorer des frontières. Et c'est encore le rapport ainsi établi entre activité et intelligence qui explique que la constitution de la société religieuse soit incomparablement plus lente que

\footnotetext{
${ }^{6}$ S. 4, 322. L'idée se trouvait déjà dans le Cours qui soulignait la «tendance fondamentale [de la vie industrielle] à lier directement tous les peuples» en vertu de «son évidente propriété exclusive de comporter et même de provoquer la simultanéité la plus étendue, tandis que, dans l'essor militaire, l'activité des uns suppose ou détermine la compression nécessaire des autres » (C. 56 ${ }^{\mathrm{e}} 1$, dans Physique sociale, Hermann, 1975, 501).
} 
celle du marché mondial. Ils ne font pas appel aux mêmes mobiles. Si l'Église est la plus étendue des sociétés, c'est aussi la moins complète, en ce sens qu'elle repose sur ce qu'il y a de moins énergique en nous.

La nature intellectuelle du lien religieux. Expliquer à quelle complétude il est ici fait allusion demanderait un exposé de la seconde philosophie de Comte qui ne pourrait qu'ennuyer le lecteur. Qu'il suffise de retenir que le terme renvoie à un ensemble de tripartitions omniprésentes dans les écrits de cette période : c'est ainsi que famille, cité et église sont données comme fondées respectivement sur l'affectivité (le cœur), l'activité (le caractère) et sur l'intelligence (l'esprit) ou que la devise ordre et progrès cède la place à : l'amour pour principe, l'ordre pour base et le progrès pour but. Or il n'y a pas d'idée plus constante chez Comte que celle de la faiblesse de notre intelligence, doublement subordonnée à l'activité puis à l'affectivité ; d'où cette remarque que l'on ne s'attend pas à trouver sous sa plume : on se lasse de penser, on se lasse même d'agir, on ne se lasse pas d'aimer. Mais que l'esprit soit la moins énergique de nos facultés ne signifie pas qu'il n'ait aucun rôle à jouer. Présenter la société religieuse comme la moins complète est ainsi une façon détournée d'en souligner le caractère avant tout intellectuel : l'église est une communauté de croyants, elle se définit par son credo.

L'approche est quelque peu inhabituelle en ce sens que l'on s'attache plus volontiers d'ordinaire au fondement irrationnel de la religion; mais la foi qui unira les croyants est une foi positive. Les dogmes de la nouvelle religion ne font que reprendre les principaux acquis de la science. Ils sont donc toujours démontrables, ce qui garantit l'universalité de la société religieuse : tout homme normalement constitué ne peut que leur donner son assentiment. Cette extension supérieure tient encore à ce que les idées ont en propre de se jouer des frontières : si on peut faire obstacle à la libre circulation des biens et des personnes, on voit mal par quels moyens on pourrait empêcher les idées de circuler.

C'est cette seconde caractéristique qui permet à la société religieuse de remplir ses fonctions. Ses capacités de guide spirituel en découlent immédiatement; quant aux deux autres, elles sont complémentaires, lutter contre les abus du pouvoir n'ayant de sens que si l'on est prêt à en consacrer l'usage légitime. De ce que le pouvoir temporel repose en dernière instance sur la force, il s'ensuit qu'il court sans cesse le risque d'être renversé par la force ; d'où le besoin de trouver un assentiment volontaire. Si la science est appelée à devenir la base spirituelle de l'ordre social, c'est qu'une société digne de ce nom repose sur l'acceptation commune d'une même vérité. Ceci vaut notamment de la vie économique : les mécanismes du marché sont régis par des forces aveugles et c'est pourquoi il importe de compléter cette association imposée par la contrainte par une association librement consentie.

L'Europe fournit un excellent exemple de cette situation. Comte y voyait en effet la première étape vers l'avènement de la société planétaire qu'il avait conçue. A une époque où les penseurs étaient obnubilés par le problème des nationalités, lui et ses disciples ont cherché, sans succès, à mettre en place une République occidentale. Les impasses dans lesquelles se trouve aujourd'hui la construction européenne redonnent une certaine actualité à ce qui a longtemps passé pour une excentricité supplémentaire de la politique positive ${ }^{7}$. L'Union européenne est née comme un marché commun mais les limites d'une telle approche sont de plus en plus manifestes et il est clair que l'issue se trouve dans une autre direction. Quoi qu'en aient les eurosceptiques, le repli sur soi ne mène nulle part. Le constat souvent fait d'un déficit démocratique ne désignerait-il pas, en creux, l'absence de ce nouveau type de lien social que Comte nous proposait d'instaurer?

\footnotetext{
${ }^{7}$ Voir la mise au point de Lévi-Strauss dans Auguste Comte et l'Italie, Commentaire ${ }^{\circ}$ 143/Automne 2013.
} 
Ces quelques tentatives pour faire sens de la théorie positive de la religion n'ont certainement pas comblé le fossé qui sépare celle-ci de ce que nous pouvons constater autour de nous. Aujourd'hui comme il y a cent cinquante ans, tout cela reste vue de l'esprit sans grande prise sur la réalité. Que le but soit encore éloigné, qui le niera ? La question est plutôt de savoir si la direction est bonne ou non. La décomposition des croyances théologiques semble un processus irréversible et on comprend de moins en moins comment un être raisonnable peut y adhérer; mais rien n'autorise à conclure, comme on le fait d'ordinaire, de la mort de Dieu à la disparition des religions. Tôt ou tard, il nous faudra apprendre à dissocier religion et théologie, ainsi que nous y invite Comte. Comme le remarque A. de Botton dans son Guide des religions à l'usage des mécréants,

notwithstanding its many oddities, Comte's religion is hard to dismiss out of hand, for it identified important fields in atheistic society that continue to lie fallow and to invite cultivation and showed a pioneering interest in generating institutional support for ideas. His ability to sympathise with the ambitions of traditional religions, to study their methods and to adapt them to the needs of the modern word reflected a level of creativity, tolerance and inventiveness to which few latter critics of religion have been capable of rising 8 .

Il a fallu des millénaires à l'humanité pour passer de la société domestique à la société politique, pourquoi le passage à la société religieuse devrait-il se faire en quelques siècles ?

\footnotetext{
${ }^{8}$ Alain de Botton : Religion for Atheists, Penguin, 2012, 306-307.
} 